\title{
Stability Property for the Predator-Free Equilibrium Point of Predator-Prey Systems with a Class of Functional Response and Prey Refuges
}

\author{
Fengde Chen, Yumin Wu, and Zhaozhi Ma \\ College of Mathematics and Computer Science, Fuzhou University, Ujian, Uzhou 350002, China \\ Correspondence should be addressed to Fengde Chen, fdchen@fzu.edu.cn
}

Received 30 January 2012; Accepted 13 March 2012

Academic Editor: Vimal Singh

Copyright (c) 2012 Fengde Chen et al. This is an open access article distributed under the Creative Commons Attribution License, which permits unrestricted use, distribution, and reproduction in any medium, provided the original work is properly cited.

We investigate the stability property for the predator-free equilibrium point of predatorprey systems with a class of functional response and prey refuges by using the analytical approach. Under some very weakly assumption, we show that conditions that ensure the locally asymptotically stable of the predator-free equilibrium point are consistent with that of the globally asymptotically stable ones. Our result supplements the corresponding result of Ma et al., 2009.

\section{Introduction}

Stability analysis of a predator-prey system incorporating a given functional response becomes a popular issue in mathematical ecology during the last decade [1-10]. Ma et al. [10] considered the effects of prey refuges on a predator-prey model with a class of functional response, for example,

$$
\begin{gathered}
\dot{X}=r X\left(1-\frac{X}{K}\right)-p \varphi(X-\beta X) Y \\
\dot{Y}=(q \varphi(X-\beta X)-d) Y
\end{gathered}
$$

where $X(t)$ and $Y(t)$ denote the density of prey and predator populations at time $t$, respectively. The term $\varphi(X)$ represents the functional response of the predator population and satisfies the following assumption:

$$
\varphi(0)=0, \quad \varphi^{\prime}(X)>0 \quad(X>0) .
$$


By using the change of variables:

$$
\phi:\left(R_{0}^{+}\right)^{2} \longrightarrow\left(R_{0}^{+}\right)^{2}, \quad \phi(X, Y)=\left(\frac{x}{1-\beta},(1-\beta) y\right)
$$

system (1.1) is equivalent to the following model:

$$
\begin{gathered}
\dot{x}=r x\left(1-\frac{x}{(1-\beta) K}\right)-p \varphi(x) y, \\
\dot{y}=(q \varphi(x)-d) y .
\end{gathered}
$$

Concerned with the stability property of the predator-free equilibrium, by analysing the Jacobian matrix, the authors obtained the following conclusions.

Conclusion 1. $E_{1}((1-\beta) K, 0)$ is locally asymptotically stable if and only if $q \varphi((1-\beta) K)-d<0$.

Based on this conclusion, without any other deduction, they declared (see [10, Theorems 4.1(3) and 4.2(3)]).

Conclusion 2. If $1-\varphi^{-1}(d / q) / K<\beta<1$, then predator goes extinct while prey population reaches its maximum environment carrying capacity.

Note that Conclusion 1 is local one while Conclusion 2 reflects the globally property of the system (1.4). Obviously there is a gap between these two conclusions. To show the Conclusion 1 implies Conclusion 2, some more detail analysis is needed. The aim of this paper is try to show that under some very weakly assumption on $\varphi$, the local asymptotical stability of the predator-free equilibrium point do implies the global ones. More precisely, we obtain the following result.

Theorem 1.1. Assume that $\varphi(x)=\varphi_{1}(x) x$ and there exists a positive constant $L$ such that $\varphi_{1}(x) \leq L$ for all $x>0$ holds, assume further that $1-\varphi^{-1}(d / q) / K<\beta<1$ holds. Then predator species will be extinct while prey population reaches its maximum environment carrying capacity.

Remark 1.2. Many explicit forms for the predator functional response that have been used are satisfy above assumption. For example,

$$
\begin{aligned}
& \frac{b x}{a+x} \text { [Holling type II], } \\
& \frac{b x^{2}}{a+x^{2}}[\text { Holling type III], } \\
& a(1-\exp \{-c x\})[\text { Ivlev], } \\
& b x^{\gamma}, \quad 0<\gamma<1 \text { [Rosenzweig]. }
\end{aligned}
$$

\section{Proof of the Main Result}

Proof. We first show that under the assumption of Conclusion 2, predator species will be driven to extinction. 
It follows from $1-\varphi^{-1}(d / q) / K<\beta<1$ and the continuity of $\varphi$, for enough small positive constant $\varepsilon$, the following inequality holds:

$$
\beta>1-\frac{\varphi^{-1}((d-\varepsilon) / q)}{K+\varepsilon},
$$

that is,

$$
\varphi^{-1}\left(\frac{d-\varepsilon}{q}\right)>(1-\beta)(K+\varepsilon)
$$

Since $\varphi^{\prime}(X)>0, X>0$, inequality (2.2) is equal to the following inequality:

$$
d-\varepsilon>q \varphi((1-\beta)(K+\varepsilon)) .
$$

From the first equation of system (1.4), we have

$$
\dot{x} \leq r x\left(1-\frac{x}{(1-\beta) K}\right) .
$$

Therefore,

$$
\limsup _{t \rightarrow+\infty} x(t) \leq(1-\beta) K
$$

For $\varepsilon$ defined by (2.1), inequality (2.5) shows that there exists an enough large $T$ such that

$$
x(t)<(1-\beta)(K+\varepsilon), \quad \forall t \geq T .
$$

And so, for $t \geq T$, from the second equation of system (1.4) and (2.3), one has

$$
\dot{y} \leq(q \varphi((1-\beta)(K+\varepsilon))-d) y<-\varepsilon y .
$$

That is,

$$
y(t) \leq y(T) \exp \{-\varepsilon(t-T)\} \longrightarrow 0, \quad \text { as } t \longrightarrow+\infty
$$

For any small positive constant $\varepsilon_{1}>0$ which satisfies $\varepsilon_{1} \leq r / 2 p \mathrm{~L}$, there exists a $T_{1}>T$ such that

$$
y(t) \leq \varepsilon_{1}, \quad \forall t>T_{1}
$$


On the other hand, since $\varphi(x)=\varphi_{1}(x) x$ and $\varphi_{1}(x) \leq L$ for all $x>0$. Equation (2.9) together with the first equation of (1.4) leads to

$$
\begin{aligned}
\dot{x} & =r x\left(1-\frac{x}{(1-\beta) K}\right)-p \varphi_{1}(x) x y \\
& \geq r x\left(1-\frac{x}{(1-\beta) K}\right) x-p L \varepsilon_{1} x \\
& \geq r x\left(\frac{1}{2}-\frac{x}{(1-\beta) K}\right)
\end{aligned}
$$

for all $t \geq T_{1}$. From this different inequality, one could easily obtain that,

$$
\liminf _{t \rightarrow+\infty} x(t) \geq \frac{(1-\beta) K}{2}
$$

Now we introducing a transformation $z=x-(1-\beta) k$, then the first equation of system (1.4) is equivalent to

$$
\dot{z}=-\frac{r z}{(1-\beta) k}(z+(1-\beta) k)-p \varphi(z+(1-\beta) k) y .
$$

From (2.5), (2.11), and (2.12) we know that $z(t)$ is bounded differentiable on $(0, \infty)$, Let $\bar{z}=\lim \sup _{t \rightarrow+\infty} z(t), \underline{z}=\lim \inf _{t \rightarrow+\infty} z(t)$, According to Fluctuation lemma [11], there exists sequences $\tau_{n} \rightarrow \infty, \sigma_{n} \rightarrow \infty$ such that $\dot{z}\left(\tau_{n}\right) \rightarrow 0, \dot{z}\left(\sigma_{n}\right) \rightarrow 0, z\left(\xi_{n}\right) \rightarrow \underline{z}$ and $z\left(\sigma_{n}\right) \rightarrow \bar{z}$. Also, it follows from (2.12) that

$$
\begin{gathered}
\dot{z}\left(\sigma_{n}\right)=-\frac{r z\left(\sigma_{n}\right)}{(1-\beta) k}\left(z\left(\sigma_{n}\right)+(1-\beta) k\right)-p \varphi\left(z\left(\sigma_{n}\right)+(1-\beta) k\right) y\left(\sigma_{n}\right) . \\
\dot{z}\left(\xi_{n}\right)=-\frac{r z\left(\xi_{n}\right)}{(1-\beta) k}\left(z\left(\xi_{n}\right)+(1-\beta) k\right)-p \varphi\left(z\left(\xi_{n}\right)+(1-\beta) k\right) y\left(\xi_{n}\right) .
\end{gathered}
$$

Since (2.11) implies that

$$
\lim _{n \rightarrow+\infty}\left(z\left(\xi_{n}\right)+(1-\beta) k\right) \geq \frac{(1-\beta) K}{2}
$$

Taking limit in (2.13) and (2.14), it follows from (2.8) and (2.16) that

$$
0=\bar{z}=\underline{z}
$$

that is

$$
\lim _{t \rightarrow+\infty} z(t)=0
$$


which is equivalent to say that

$$
\lim _{t \rightarrow+\infty} x(t)=(1-\beta) K
$$

This ends the proof of Theorem 1.1.

\section{Acknowledgments}

Research was supported by the Natural Science Foundation of Fujian Province (2011J01007) and the Technology Innovation Platform Project of Fujian Province (2009J1007).

\section{References}

[1] T. K. Kar, "Stability analysis of a prey-predator model incorporating a prey refuge," Communications in Nonlinear Science and Numerical Simulation, vol. 10, no. 6, pp. 681-691, 2005.

[2] T. K. Kar, "Modelling and analysis of a harvested prey-predator system incorporating a prey refuge," Journal of Computational and Applied Mathematics, vol. 185, no. 1, pp. 19-33, 2006.

[3] P. D. N. Srinivasu and I. L. Gayatri, "Influence of prey reserve capacity on predator-prey dynamics," Ecological Modelling, vol. 181, no. 2-3, pp. 191-202, 2005.

[4] W. Ko and K. Ryu, "Qualitative analysis of a predator-prey model with Holling type II functional response incorporating a prey refuge," Journal of Differential Equations, vol. 231, no. 2, pp. 534-550, 2006.

[5] Y. Huang, F. Chen, and L. Zhong, "Stability analysis of a prey-predator model with Holling type III response function incorporating a prey refuge," Applied Mathematics and Computation, vol. 182, no. 1, pp. 672-683, 2006.

[6] F. Chen, L. Chen, and X. Xie, "On a Leslie-Gower predator-prey model incorporating a prey refuge," Nonlinear Analysis. Real World Applications, vol. 10, no. 5, pp. 2905-2908, 2009.

[7] Y. Tao, X. Wang, and X. Song, "Effect of prey refuge on a harvested predator-prey model with generalized functional response," Communications in Nonlinear Science and Numerical Simulation, vol. 16, no. 2, pp. 1052-1059, 2011.

[8] L. Chen, F. Chen, and L. Chen, "Qualitative analysis of a predator-prey model with Holling type II functional response incorporating a constant prey refuge," Nonlinear Analysis. Real World Applications, vol. 11, no. 1, pp. 246-252, 2010.

[9] L. Ji and C. Wu, "Qualitative analysis of a predator-prey model with constant-rate prey harvesting incorporating a constant prey refuge," Nonlinear Analysis. Real World Applications, vol. 11, no. 4, pp. 2285-2295, 2010.

[10] Z. Ma, W. Li, Y. Zhao, W. Wang, H. Zhang, and Z. Li, "Effects of prey refuges on a predator-prey model with a class of functional responses: the role of refuges," Mathematical Biosciences, vol. 218, no. 2, pp. 73-79, 2009.

[11] F. Montes de Oca and M. Vivas, "Extinction in two dimensional Lotka-Volterra system with infinite delay," Nonlinear Analysis. Real World Applications, vol. 7, no. 5, pp. 1042-1047, 2006. 


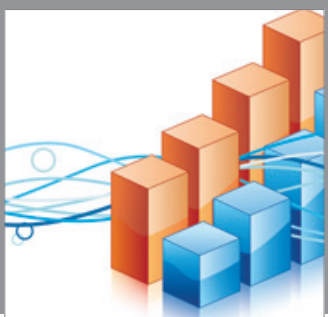

Advances in

Operations Research

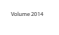

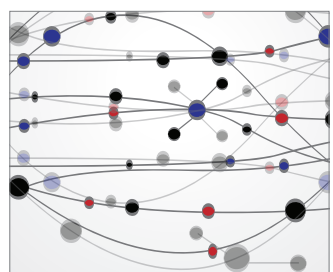

\section{The Scientific} World Journal
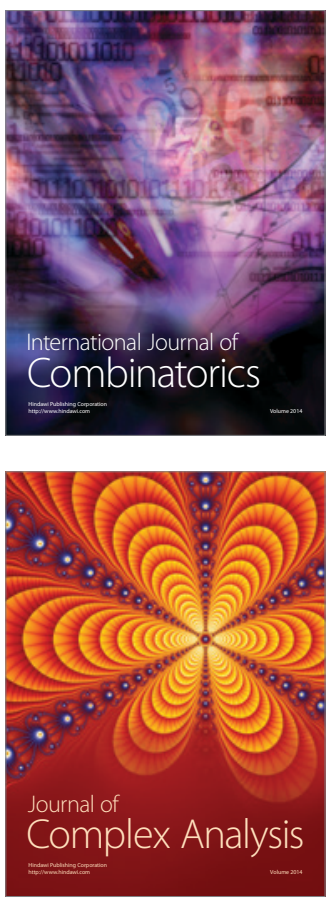

International Journal of

Mathematics and

Mathematical

Sciences
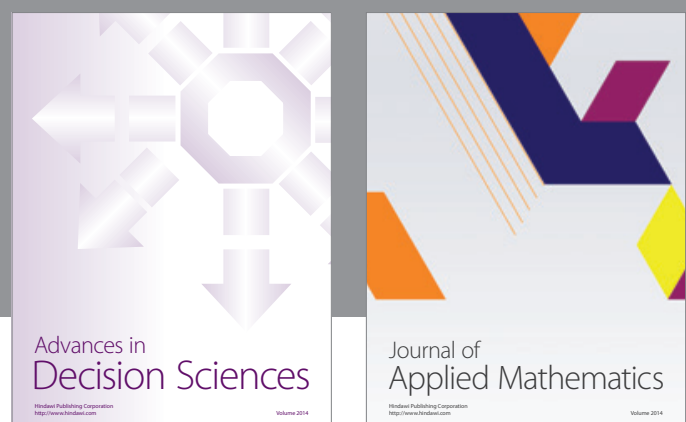

Journal of

Applied Mathematics
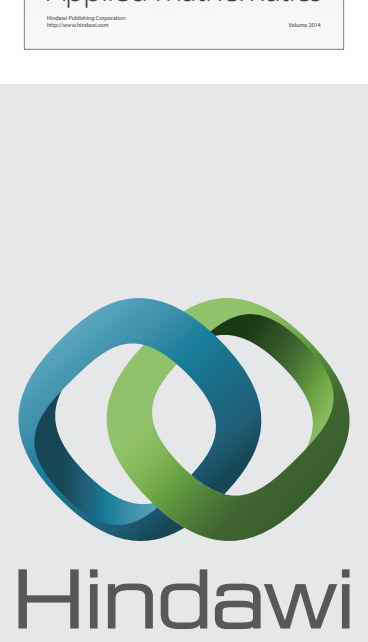

Submit your manuscripts at http://www.hindawi.com
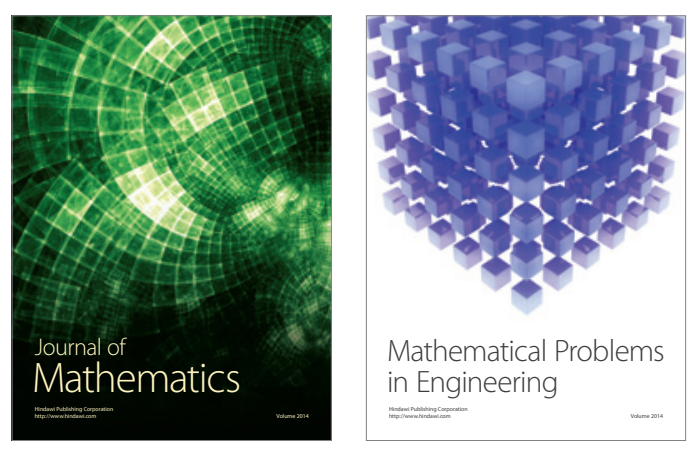

Mathematical Problems in Engineering
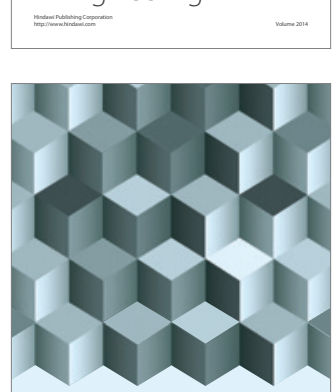

Journal of

Function Spaces
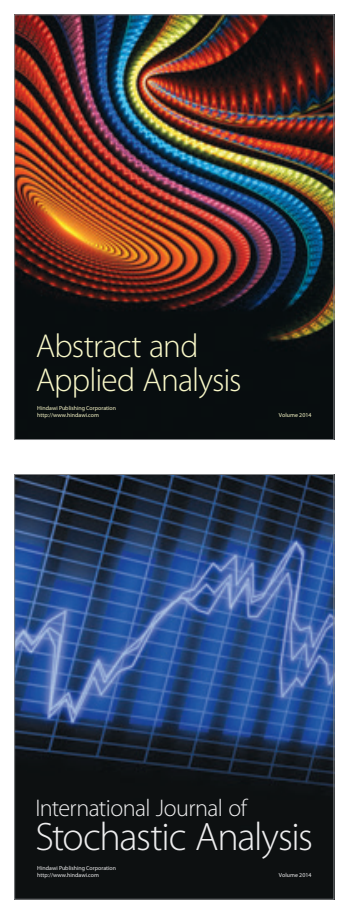

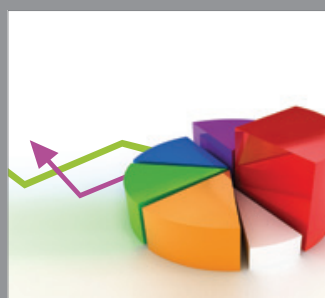

ournal of

Probability and Statistics

Promensencen
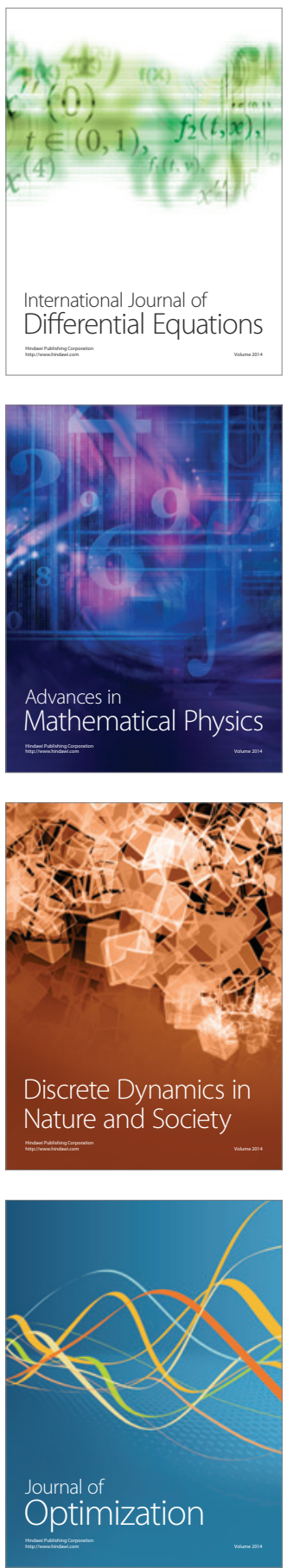